\title{
Reconstrucción Tridimensional de Superficies en el Cuerpo Humano
}

\author{
Jérica Risent, Jaime Masiá*, Juan López y Santiago Ferrándiz \\ Departamento Ingeniería Mecánica y de Materiales, Universidad Politécnica de Valencia. \\ Plaza Ferrándiz y Carbonell, s/n. 03801 Alcoy (Alicante), España (e-mail: jerirod1@epsa.upv.es, \\ jmasia@mcm.upv.es, jlopezm@mcm.upv.es, sferrand@mcm.upv.es)
}

* Autor a quien debe ser dirigida la correspondencia

Recibido Mar. 13, 2013; Aceptado Abr. 29, 2013; Versión final recibida Jun. 11, 2013

\section{Resumen}

Se presenta un estudio del proceso de alineación de datos obtenidos de morfologías humanas mediante un dispositivo de adquisición por láser. Se utiliza un dispositivo digitalizador tridimensional de última generación y se establece una metodología de trabajo para el proceso de alineación de los datos obtenidos de morfologías humanas. Además, se indican las directrices para resolver los problemas derivados de esta alineación y se realiza el estudio comparativo de programas de tratamiento de nubes de puntos. RapidForm ${ }^{\mathrm{TM}}$, el software estándar utilizado para la edición de los datos adquiridos por el escáner 3D es comparado con Polyworks ${ }^{\mathrm{TM}}$, el software alternativo a RapidForm ${ }^{\mathrm{TM}}$, debido a su calidad de alineamiento de piezas mecánicas. Resultados de la comparación de algunas aplicaciones de los programas son presentados y discutidos.

Palabras clave: reconstrucción 3D, morfologías humanas, alineación de datos, RapidForm, Polyworks

\section{Three-dimensional reconstruction of surfaces in human body}

\begin{abstract}
A study on the alignment process of data of human morphology obtained by laser acquisition devices is presented in this paper. A three-dimensional latest generation digitizer is used and a methodology of alignment for the human morphology data is established. Also, guides on how to solve problems of this alignment are discussed and a comparative study of point clouds processing programs is carried out. RapidForm ${ }^{\mathrm{TM}}$, the standard software used for editing data acquired by the scanner 3D is compared with Polyworks ${ }^{\mathrm{TM}}$, the alternative to RapidForm ${ }^{\mathrm{TM}}$ software used due to its good characteristics for the alignment mechanical parts. Results of the comparison of some software applications are showed.
\end{abstract}

Keywords: 3D reconstruction, cloud points, scanner, human morphology, data alignment, RapidForm, Polyworks 


\section{INTRODUCCIÓN}

La digitalización tridimensional es una tecnología que, desde sus inicios como herramienta de verificación, ha sufrido grandes transformaciones. Pero ha sido a partir de los últimos años cuando ha experimentado la evolución más profunda. Uno de los elementos que ha permitido realizar este progreso ha sido la disminución de costes en los equipos de digitalización (Yang y Medioni, 1991; Bernardini y Rushmeier, 2002) así como en los elementos de computación y todos los dispositivos electrónicos que están relacionados con la captura digital. También es conocido que en una gran cantidad de casos existe un modelo desarrollado mediante un sistema CAD. En otros no existe dicho modelo y por lo tanto debe recurrirse a la captura de la información pertinente desde los propios modelos físicos. Para confeccionar la construcción de los modelos deben seguirse una serie de pasos (Yang y Medioni, 1991; Mahadevan et al., 2001; Pahole et al., 2005): adquisición de datos, que además pueden ser caracterizados como sistemas activos y pasivos; archivo de vistas, que contempla la manipulación de datos con objeto de alinear las nubes de puntos; reconstrucción de las superficies. Estas reconstrucciones se realizan a dos niveles, el volumétrico y el geométrico. En este último punto Chang y Chang (2002) ya explica que en la digitalización se obtienen gran cantidad de coordenadas en 3D y que éstas deben utilizarse para realizar la reconstrucción de las superficies. Por ello se recurre a una serie de medidas que permitan una reducción de estos puntos y que de alguna manera provocan una reorganización de la información y una optimización de la misma. Por su parte Remondino (2003) establece una clasificación de las técnicas de reconstrucción de las superficies en 3D. Subdivide los métodos en sistemas basados en medidas de objetos y sistemas que no emplean medidas.

Estas técnicas (basadas en imágenes o en los sensores activos 3D) se pueden dividir principalmente en dos categorías. En la primera se habla de métodos basados en la triangulación que utilizan las medidas de la imagen, luz estructurada, luz cifrada (Cuesta, 2008). En la segunda se habla de métodos que no requieren correspondencias como las que estiman los normales de las superficies en vez de los datos 3D. Los ejemplos son la forma de sombrear, forma de la textura, forma especular, forma del contorno, forma a partir de los gradientes del borde en 2D.En los sistemas que no emplean medidas, la generación tridimensional se realiza mediante elementos simples, similar a lo que serían cajas poligonales.

Para la reconstrucción de superficies, existen diversas clasificaciones de los algoritmos empleados (Remondino, 2003). La primera clasificación se podría realizar de acuerdo con la calidad de los puntos capturados. El primer algoritmo sería el denominado como nube de puntos desorganizada (Hope et al., 1992; Mencl, 2001). Estos algoritmos se caracterizan por trabajar sobre datos desorganizados los cuales no contienen ningún tipo de información salvo su posición espacial. Dado que no existe ninguna información, nos obliga a realizar la reconstrucción de acuerdo con su coherencia. A continuación podría hablarse de las nubes de puntos estructuradas. En estos casos sí se dispone de información adicional de cada uno de los puntos capturados.

Puede realizarse otro tipo de clasificación de acuerdo con el tipo de representación de la superficie, quedando esta clasificación como representación paramétrica, en la que destacan las representaciones de b-spline, curvas bezier, representación implícita, en la que se intenta alcanzar una función implícita que se encargará de evaluar un determinado valor. Completan esta clasificación tres algoritmos denominados como representación sencilla, la que se caracteriza por la representación superficial como una entidad simple compuesta por puntos, bordes y triángulos; el algoritmo de superficies aproximadas (Jiao y Edelsabrunner, 1999) en las que no se trabaja sobre todos los puntos originales, pero sí sobre los puntos más cercanos posibles y el último algoritmo es el de las superficies interpoladas (Giesen y Dey, 2001) en las que se trabaja sobre nubes de puntos que generan modelos muy precisos y en la que se respeta una correcta conexión de datos.Otra clasificación que aparece (Remondino, 2003) es la que se realiza de acuerdo con la subdivisión espacial. El primer algoritmo (Hope et al., 1992) queda definido como el basado en orientación de superficies, el cual no distingue entre superficies cerradas o abiertas. La gran mayoría de algoritmos desarrollados se enmarcan en este grupo. El segundo algoritmo se define como de volúmenes orientados que trabaja siempre sobre superficies cerradas, generalmente basado en tetraedros.

La aplicación de la digitalización a morfologías no analíticas como son las del cuerpo humano, abre un gran abanico de posibilidades en la obtención de información para el diseño de productos personalizados. En estos casos se han llegado a aplicar determinados algoritmos desarrollados por Remondino y Roditakis (2003). En ellos se han empleado ecuaciones colineares que correlacionan los puntos espaciales con las proyecciones de los mismos sobre la imagen en 2D. La tendencia no solo es la aplicación a productos personalizados, sino otras líneas de trabajo como pueden ser el reconocimiento de patrones humanos 0 patrones biométricos (Heisele y Poggio, 2003; Mori et al., 2005) como son los empleados en los programas utilizados en el presente artículo para realizar el alineamiento de las superficies. 
Como objetivo final del trabajo, se pretende por tanto estudiar como aplican los programas empleados estos algoritmos de forma que se pueda determinar la idoneidad de los mismos en el proceso de alineamiento de superficies tan complejas como son las del cuerpo humano.

\section{EXPERIMENTAL}

Para los trabajos de laboratorio se ha empleado un escáner VIVID-910 ${ }^{\mathrm{TM}}$ (fabricado por Konica-Minolta) que utiliza tecnología láser en la captura de geometrías tridimensionales y su imagen a color en 0.3 segundos. Se pueden digitalizar volúmenes (entre $110 \times 80 \times 40 \mathrm{~mm}^{3}$ y $1200 \times 900 \times 400 \mathrm{~mm}^{3}$ ) mediante las lentes intercambiables. La resolución de los datos que se obtienen al realizar el escaneado es lo suficientemente buena para utilizarlos en aplicaciones industriales, medicina, realidad virtual, reconstrucción cultural y campos de investigación. Está dotado de una pantalla CCD de 640 × 480 píxeles y cuatro filtros rotatorios (para R, G, B y mediciones 3D). El modo de escaneado se puede establecer en modo FAST (se utiliza cuando la velocidad de escaneado es importante) y modo FINE (se utiliza cuando la precisión de datos es importante). Según el manual de instrucciones del escáner, el modo FAST se utiliza para objetos como el cuerpo humano, que se mueven durante el proceso de adquisición. Así cada modo presenta las siguientes características: Modo FAST: $0.3 \mathrm{~s}$. con una resolución de puntos de información de $320 \times 240$. (76.800 puntos de información). Modo FINE: 2.5 s. con una resolución de puntos de información de 640 x 480 . (307.200 puntos de información).

Polygon Editing Tool (PET) es el software que utiliza el escáner VIVID910 TM para la adquisición de datos 3D. En él se pueden configurar los parámetros del escaneado, como se explica a continuación, y también se pueden realizar operaciones de reparación, alineación y edición de los datos adquiridos. Estas operaciones de reparación, alineación y edición también se pueden realizar con el software RapidForm ${ }^{\mathrm{TM}}$ (RF) o bien con el Polyworks ${ }^{\mathrm{TM}}$ (PW), el cual se estudiará como complementario o sustitutivo al PET, según en qué operaciones, ya que posee parámetros más precisos y realiza un mayor número de operaciones de edición.

\section{METODOLOGÍA}

La metodología empleada en el presente estudio ha quedado estructurada en 2 fases diferenciadas. En la primera fase se ha realizado la captura de datos en diversas sesiones mediante el empleo del PET, que nos ha permitido la adquisición y manipulación de los datos necesarios. La segunda etapa ha sido la edición de los datos capturados. Dentro de la etapa de edición se han aplicado tres fases de trabajo. Las siguientes fases han sido repetitivas y a la vez han servido de comparación entre los dos tipos de software (Remondino, 2003) empleado, el PW y el RF. La primera fase realizada dentro de la edición de nubes de puntos ha sido el filtrado de datos. El objetivo principal de esta fase ha sido la reparación y limpieza de cada una de las tomas efectuadas con el escáner. Finalizada esta fase, se ha confeccionado una comparativa de resultados.

La siguiente fase aplicada ha sido el alineamiento de tomas, permitiendo a su vez solapar y comprobar el conjunto de los datos con objeto de obtener una única toma general en tres dimensiones. También en esta etapa se ha confeccionado un resumen comparativo de los resultados. La última fase aplicada ha sido el cosido de las tomas para que se pueda considerar como una única superficie. Al igual que en etapas anteriores se ha confeccionado un resumen comparativo. De todos los escaneados realizados se han elegido para la alineación aquellos que se consideran de mejor calidad ya que presentan menos distorsiones y ruido en su superficie. De este modo se trata de evitar una disminución de la calidad del alineamiento debido a datos mal adquiridos.

\section{RESULTADOS Y DISCUSIÓN}

Aplicando el procedimiento de trabajo anteriormente expuesto se procede a la edición inicial de cada uno de los caparazones a los cuales pertenecen los puntos que se quiere seleccionar para editarlos posteriormente. La selección de puntos se puede realizar de diversos modos: mediante el cercado de una zona rectangular, o mediante una curva Bezier. Se utilizará una herramienta u otra dependiendo de la irregularidad del espacio a controlar. Se seleccionan en primer lugar, para su posterior eliminado, los puntos de otros objetos adquiridos en el proceso de escaneado; después, aquellos que definan caras tangentes a la definición de la superficie, así como puntos sueltos en el espacio. Dada la irregularidad de los caparazones, la mayoría de las selecciones se realizarán con la herramienta Bezier, figura 1. Cerrar el área definida por la curva Bezier se puede realizar de manera muy simple mediante la pulsación del botón derecho del ratón. En esta primera etapa los datos erróneos son eliminados (Natarajan y Edelsbrunner, 2004) lo que a su vez nos permitirá reducir el tiempo de computación posterior. 


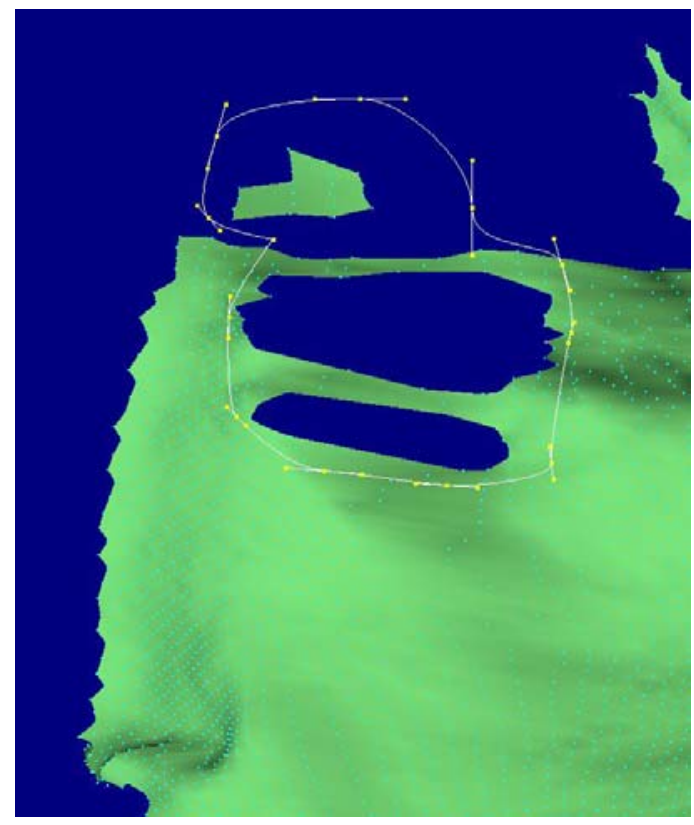

Fig. 1: Selección y eliminación de puntos y vértices.

La reparación y limpieza con el RF da comienzo con la importación de los datos obtenidos mediante el escaneado de los caparazones. Estos ficheros captados en modo FINE, contienen la malla de polígonos que definen la morfología facial y además como información adicional contienen la textura de la imagen. Esta textura es la que debe eliminarse inicialmente (Garland y Heckbert, 1998). Esta eliminación nos aporta la ventaja de un menor peso en el tamaño de fichero de datos, por lo que el manejo del tiempo de computación será menor.

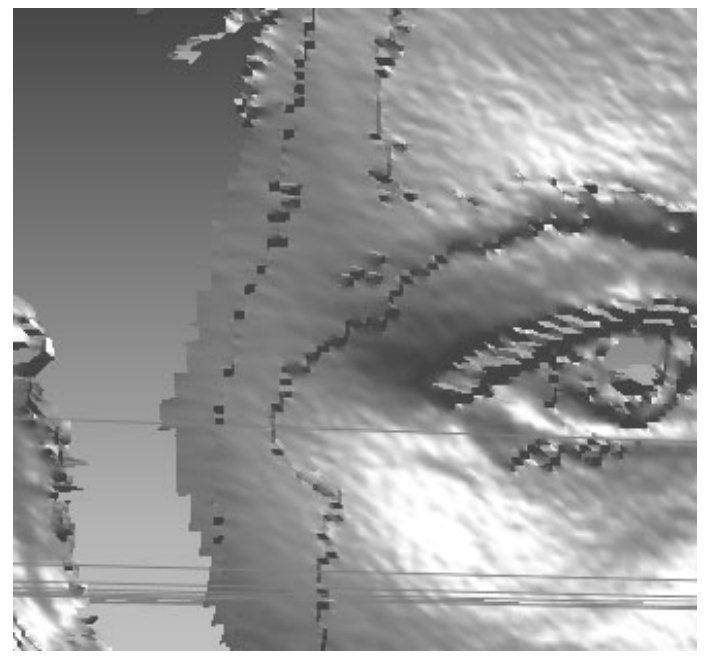

Fig. 2: Escalonado por palpitación.

El siguiente paso será la eliminación de los puntos conflictivos, figura 2, que en nuestro caso son una serie de escalones provocados por los movimientos morfológicos provocados por la respiración y que han sido captados por el escáner. Por lo tanto estos puntos se consideran defectuosos y son eliminados. Una vez realizada la limpieza, la triangulación del caparazón quedaría mostrada por la figura 3.

Para realizar la misma operación de limpieza con el PW procederemos de manera similar que con el RF. Se observan los resultados que se han obtenido en el proceso de limpieza de las tomas, figura 4.Se evidencia que en las tomas del PW se conserva mayor información. Dado que los datos iniciales son los mismos, se puede preguntar por qué sucede esto. La respuesta se encuentra en el modo de trabajo de cada uno de los programas. 


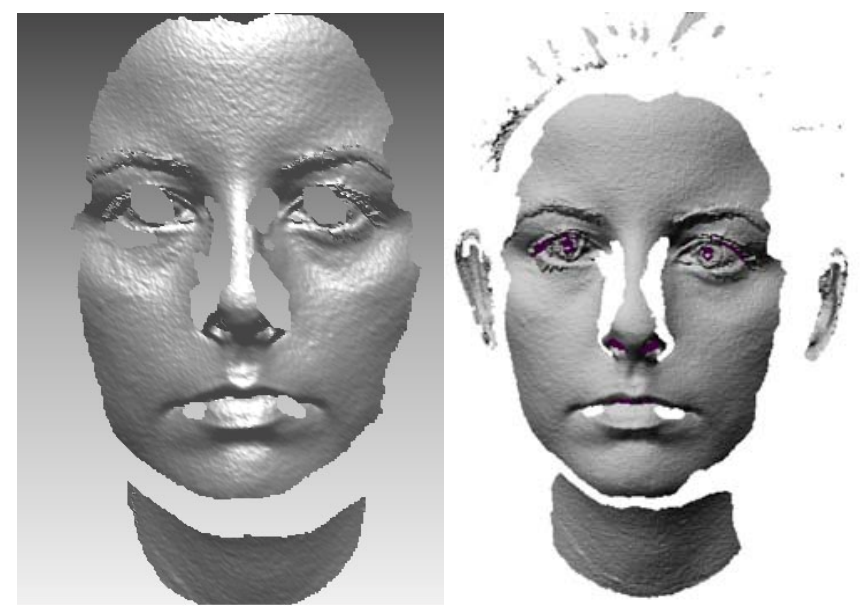

Fig. 3: Caparazón limpiado en RF y en PW.

RF
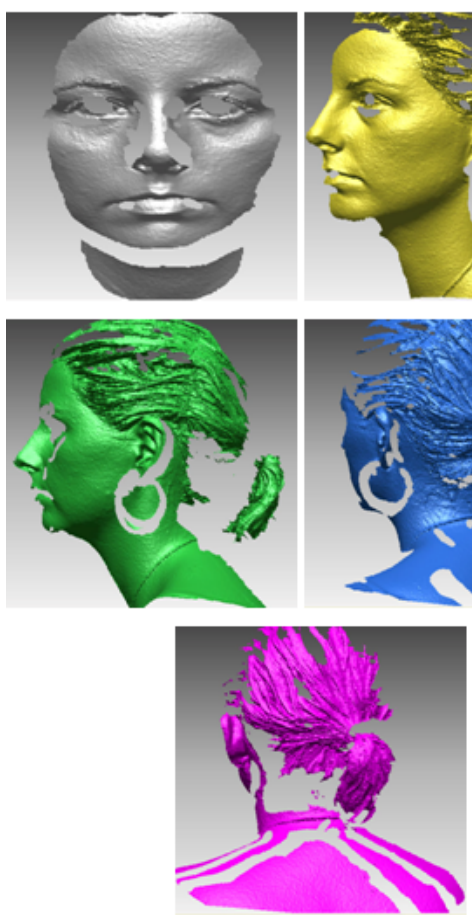

PW
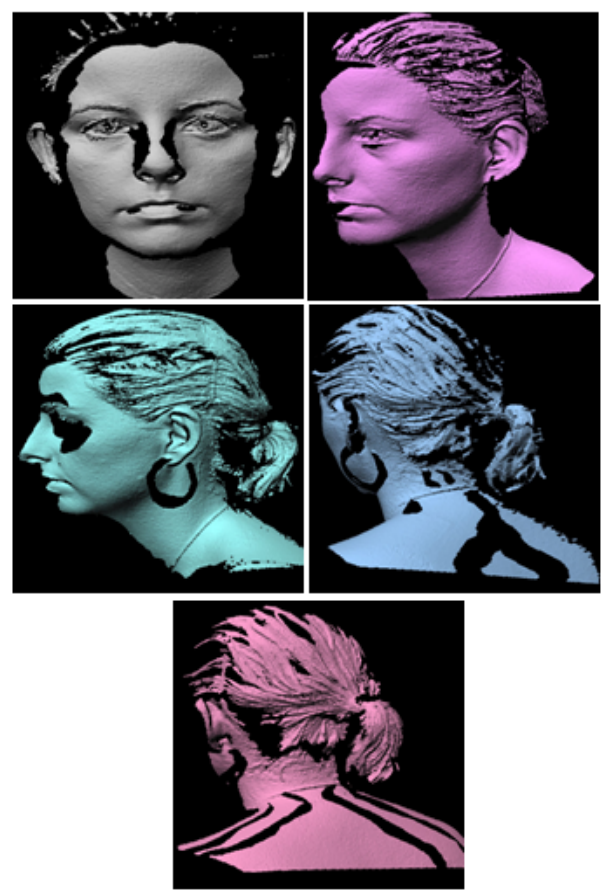

Fig. 4: Resultados de limpieza y reparación.

Cuando el RF importa un archivo *.cdm, lo triangulariza automáticamente teniendo en cuenta un parámetro que está establecido por defecto en 75 y está expresado como: "Facing Angle is Larger than...". Este dato determina cuánto de perpendiculares pueden ser las caras que se creen respecto a la vista frontal. Cuanto mayor sea el ángulo, más caras tangenciales a la superficie se tendrán. En RF este parámetro no se puede modificar a no ser que se exporten los archivos *.cdm como nubes de puntos de coordenadas, y después se poligonalice.

En PW sí se puede establecer el grado de tangencialidad en cualquier momento. Es una propiedad más de las tomas. Esto es debido a que este software maneja los archivos y sus modificaciones de modo distinto. Mantiene intactos los archivos *.cdm, y lo que hace es guardar la lista de cambios en una carpeta aparte. Así, se poseen dos carpetas: una para los archivos *.cdm originales y otra para los datos de edición que se pudieran hacer. Estas modificaciones ocupan muy poco espacio, y siempre se conserva el *.cdm original. Otra ventaja con respecto al RF, en el PW el ángulo máximo permitido para las caras más tangenciales se determinó en $80^{\circ}$. Por ello se conserva mayor información tangencial, pero no defectuosa. 
Para el proceso de alineado se efectúan los siguientes pasos: La primera toma a importar será erika1. De esta toma partirán los dos frentes de alineaciones. Un frente será el formado por los caparazones erika1, erika2, erika3, erika4 y erika5. El otro frente se compondrá de erika1, erika10, erika9, erika8 y erika7.

La toma erika6 será la que una los dos frentes de alineado. La figura 5 muestra lo que capta una toma cualquiera, como puede ser la toma erika1. Existen dos zonas en la información que se recibe que se diferencian por el tamaño de los polígonos que se forman en ellas. La zona que se muestra en gris oscuro sería la de mayor calidad: los polígonos (triángulos) que se forman por la unión de vértices tienen los lados casi de la misma longitud y definen un caparazón muy fiel al modelo escaneado. La zona que se muestra en gris claro está formada, generalmente por triángulos con los lados muy desiguales. Se forman cuando la profundidad (eje Z) varía su valor abruptamente, para una misma variación de X.
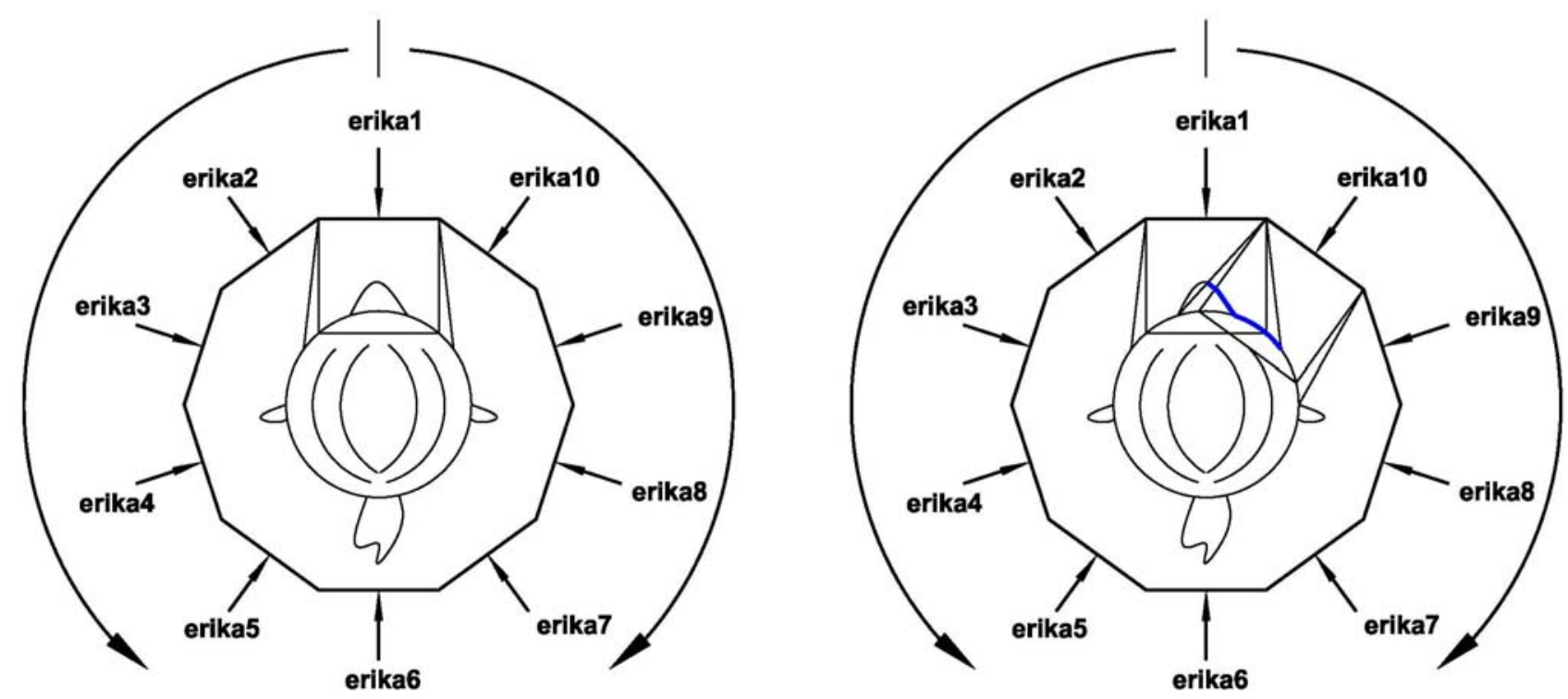

Fig. 5: Alineado de caparazones.

Para comenzar con el alineamiento de tomas con RF se deben importar todas las tomas limpias anteriormente guardadas. Las nubes de puntos y caparazones poligonales se pueden alinear usando las regiones solapadas. La alineación se lleva a cabo identificando características comunes entre los dos conjuntos de datos. Se calcula la posición aproximada de un caparazón con respecto al otro usando las características geométricas comunes. Se deben designar al menos tres pares de puntos correspondientes. El caparazón "erika1" servirá de referencia en el proceso de alineamiento. Esto quiere decir que los demás caparazones moverán su posición para alinearse con él. Para esta alineación se pueden elegir entre tres posibilidades. Éstas son la inicial, tres puntos de referencia y regional. Es conveniente el ejecutar inicialmente un alineamiento inicial que busca morfologías correspondientes. Posteriormente se pueden ejecutar los siguientes. Una vez alineados debe evaluarse la bondad de la operación, figura 6. Para ello realizaremos la medición de la desviación de la cáscara, en la que deberá introducirse la tolerancia aceptable y posteriormente realizar la medición. Como datos de salida el programa nos aportará la distancia media y la desviación estándar.

Para el caso de trabajo con el PW se procede de manera similar a lo expuesto anteriormente. Se buscan pares de puntos que permita establecer una referencia inicial. Una vez alineado también se procederá a realizar una medida de las desviaciones. La bondad de los alineamientos quedan expresados en forma de histograma, figura 7, indicándose también la desviación estándar y la media (Beraldin y Gaiani, 2003) que se ha obtenido en cada uno de los alineamientos. Los datos globales de todos los alineamientos efectuados en la presente muestra pueden observarse en la figura 8.

En la muestra 1, figura 8 las desviaciones se muestran un poco más elevadas en algunas cáscaras que en otras. Por ejemplo en el RF aparecen unas medidas más alejadas del cero en las cáscaras 6_5, 5_4 y 8_7 con respecto al PW que se muestra con mayor estabilidad. Para lo que seria la muestra 2 también se puede observar que existe una mayor desviación de un modelo respecto al otro en las cáscaras 4_3 y 1_11. En general el trato de datos con PW aparece más estable. 

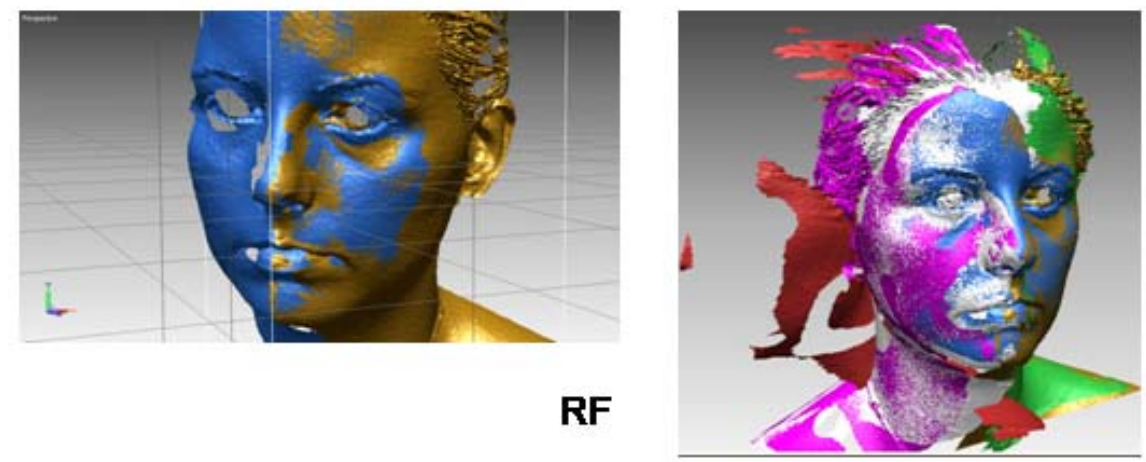

RF

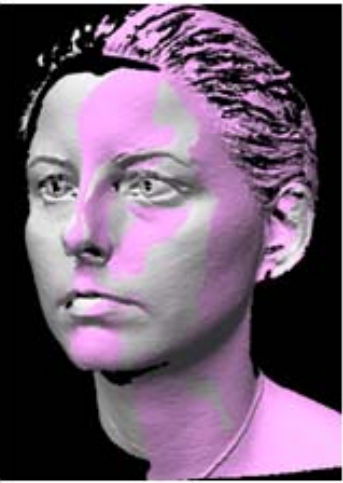

Fig. 6: Resultado de alineación RF y PW

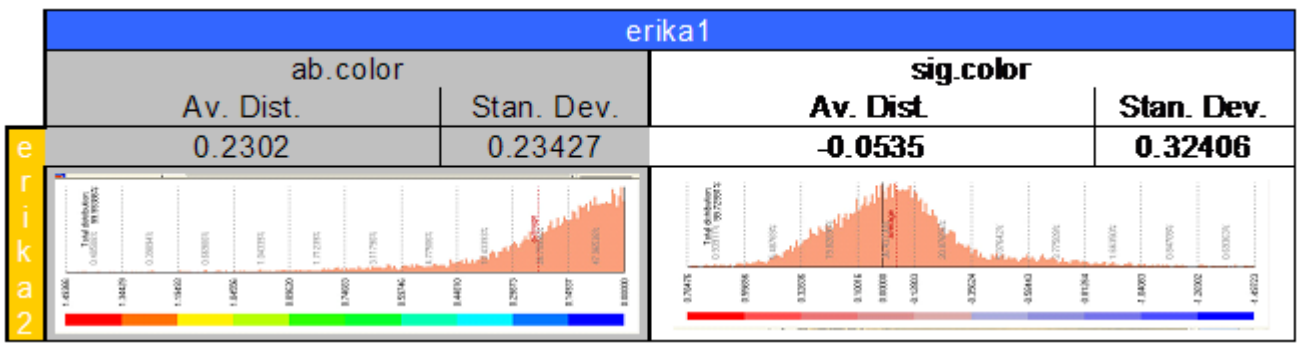

RF

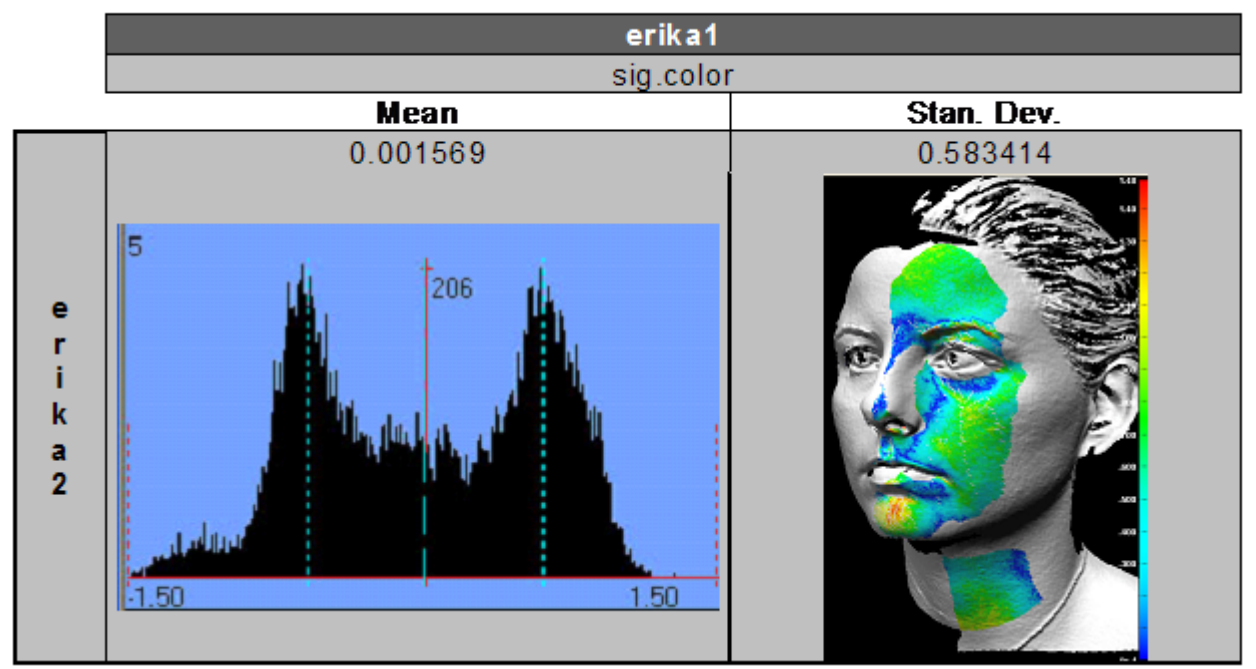

PW

Fig. 7: Representación de bondad de alineamiento 

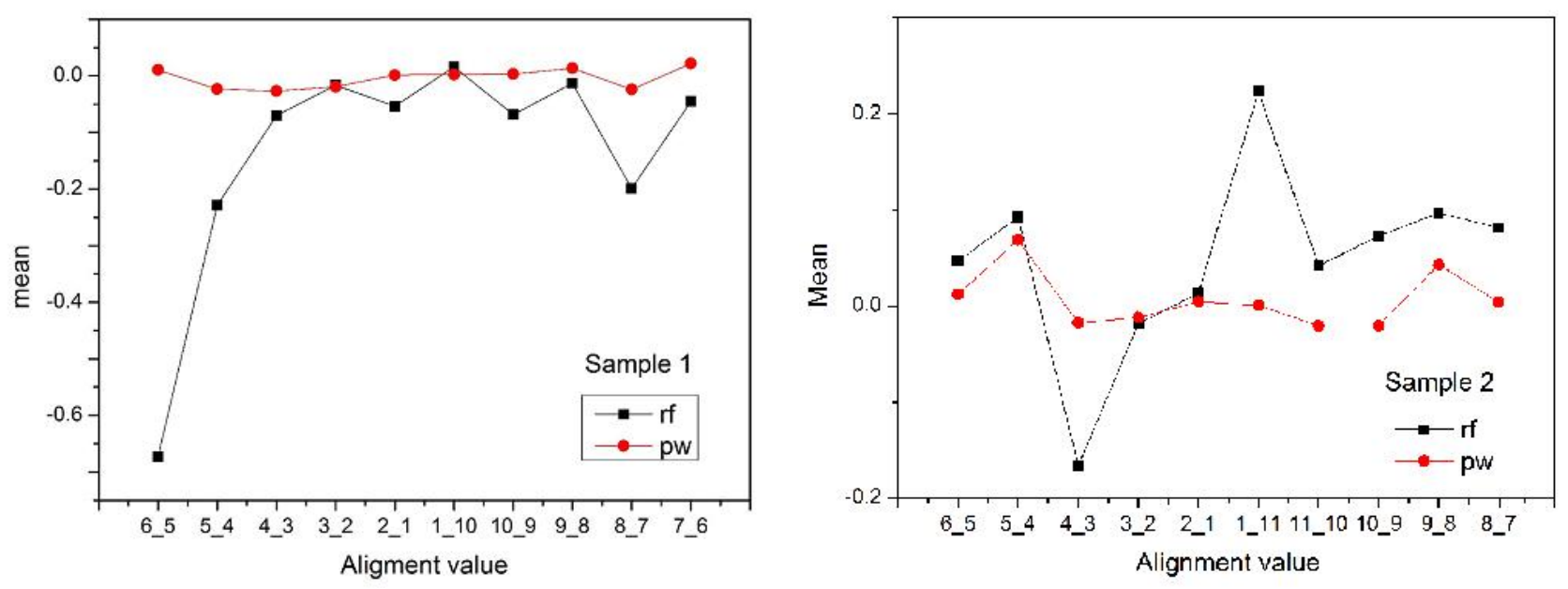

Fig. 8: Bondad de alineamientos de todas las cáscaras.
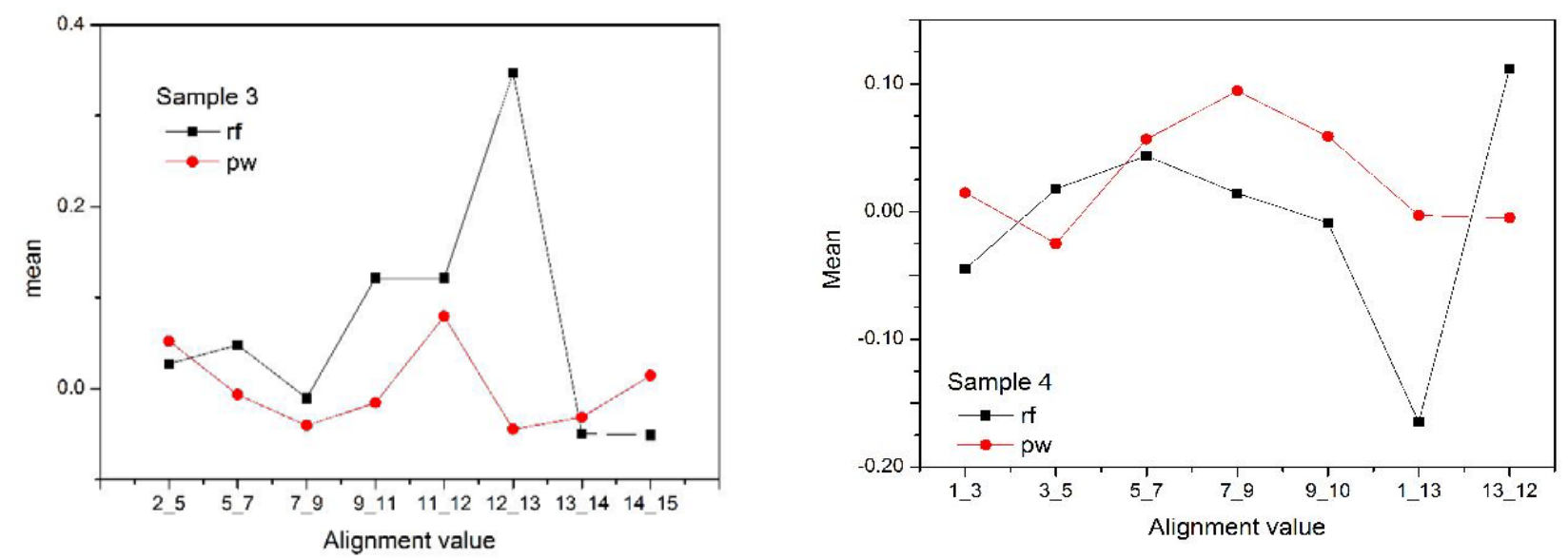

Fig. 9: Bondad de alineamiento en muestra 3 y 4.

En la muestra 3 nuevamente los puntos parecen más estables con el PW, siendo los puntos más alejados las cáscaras 11_12 y 2_5, aunque no coincide con el más alejado del RF que corresponde con la cáscara 12_13. Globalmente los puntos de la muestra 3 más estables pertenecen al tratamiento con PW. Por su parte la muestra 4 es la que aparece como la más inestable de todas. En este caso los dos programas no han tratado de una manera estable los puntos, mostrándose puntos alejados del PW en las cáscaras 5_7, 7_9 y 9_10 y en cambio el más alejado corresponde a la cáscara 1_13 con el programa RF. Desde el punto de vista de procedimientos se puede establecer la siguiente comparación:

Para el manejo de tomas el RF ofrece varias opciones de estado de tomas, como oculto y visible o bien bloqueado, desbloqueado. Con el menú emergente se pueden manejar el resto de operaciones de la toma como eliminarla, cambiar material, renombrarla o exportarla. En el PW se da la posibilidad de asignar a la capa diferentes estados como ignorada, bloqueada, liberada. Mediante menú emergente puede manejar la eliminación de toma, cambio de color, agrupar o desagrupar. Para el alineado inicial con el RF debe elegirse cual es el primer caparazón, puesto que mantiene el orden de elección, alineando el resto de cáscaras respecto al primero. Para una operativa más exacta deben configurarse los parámetros de alineamiento.

Con el PW el alineado inicial presenta un cambio de punto de vista global, lo que dificulta el manejo de las tomas en pantalla. Para un alineado más fino, se ejecuta un comando que posee los parámetros de alineado así como la visualización de los histogramas de bondad de ajuste. La verificación del ajuste en RF se realiza de forma diferente, pudiendo aplicar medidas de distancia entre puntos determinados por el usuario o bien establecer la distancia entre curvas que definan una sección transversal. Como resultados de los alineamientos se manejan precisiones del orden de décimas de milímetro, obteniéndose valores más cercanos a 0 en el PW, figura 8.

El punto final que debe abordarse en todo el procedimiento aplicado será el cosido de los caparazones. En el RF durante el proceso de unión (Merging Process) las regiones superpuestas entre los caparazones se 
eliminan. Se puede controlar el criterio de si dos caparazones se unen o no, introduciendo un valor que va de 0 a 1 . Como no se especifica en qué medida influye el valor introducido con el resultado obtenido, se realizan varias pruebas y se decide posteriormente qué criterio sería el más adecuado. El criterio aplicado finalmente ha sido 0, dado que da mejores resultados. En el PW se aplica un parámetro denominado como "step" que define el simplificado de la triangulación global. Esto sirve para regular el número de triángulos de la superficie generada. El cuadro de texto "\# Triangles" representa el número de triángulos estimado en el modelo obtenido mediante PW para un valor de "Step" dado. Se debe tener en cuenta que el algoritmo de estimación de PW generalmente sobrestima el número de triángulos en un $10 \%$. Al intentar completar el cosido han aparecido una serie de mensajes que no han permitido acabar con la operación, dado que ha existido una superposición de caparazones provocadas por los movimientos involuntarios del modelo durante el escaneado.

\section{CONCLUSIONES}

En este punto podemos establecer una serie de criterios que nos permiten concluir la comparativa. En cuanto a limpieza, el software PW proporciona nubes de datos más limpios y con menos polígonos tangenciales que el RF.

Con respecto al alineamiento con el software PW se obtienen resultados de alineamientos más precisos. En el merge o Cosido, sólo con RF es posible realizar el cosido de caparazones. Esto es debido a la acumulación de errores.

En un principio se adoptó la misma metodología que en el proceso de digitalización de piezas con superficies analíticas. A medida que se avanzó en el proceso de alineamiento fueron surgiendo una serie de problemas que con una pieza mecánica, por ejemplo, no surgirían. En el proceso de escaneado, el objeto a escanear en este caso se mueve voluntaria e involuntariamente. Voluntaria, por la respiración y el cambio de posición; involuntaria por las palpitaciones del corazón que también cambia la distancia entre dos puntos cualesquiera entre una toma y otra. Esta característica especial hace que aumente el promedio de las distancias entre puntos y que los alineamientos sean mucho menos precisos que si se tratara de una pieza mecánica.

Cambia la concepción de lo que se pretende digitalizar. No se trata de un mismo objeto del que se obtienen diferentes tomas y se alinean, y estas tomas encajan perfectamente. En realidad se trata de diferentes tomas de objetos que se parecen mucho y se pretenden alinear.

Este trabajo de comparación de las técnicas de alineamiento, permitirá poder reproducir más fielmente los modelos tridimensionales que son de aplicación en numerosos campos como la medicina, la seguridad, la juguetería, etc.

\section{REFERENCIAS}

Beraldin, J. A. y M. Gaiani, Valutazione delle prestazioni di sistemi di acquisizione tipo 3D active vision:alcuni risultati, Rivista di disegno digitale, Milan (2003).

Bernardini, F. y H. Rushmeier, The 3D model acquisition pipeline. Computer graphics forum, 21(2), 149-172 (2002).

Chang, D.Y. y Y.M. Chang, A freeform surface modelling system based on laser scan data for reverse engineering, The International Journal of Advanced Manufacturing Technology, 20, 9-19 (2002).

Cuesta, E., Fernández, P., Álvarez, B.J. y Blanco, D., Influencia del acabado superficial en el digitalizado con sensores de triangulación por láser, Información Tecnológica, 19(3), 107-114 (2008)

Garland, M. y P.S. Heckbert, Simplifying surfaces with color and texture using quadric error metrics, Proceedings of the Conference on Visualization, 263-269 (1998).

Giesen, J. y T. Dey, Detecting undersampling in surface reconstruction, Proceedings of $17^{\text {th }}$ Symposium on Computational geometry, 257-263 (2001)

Heisele, B., P. Ho, J. Wu y T. Poggio, Face recognition: component-based versus global approaches, Computer Vision and Image Understanding, 91, (2003) 
Hope, H., T. Derose, T. Duchamp, J. MacDonald y W. Stuetzle, Surface Reconstruction from unorganized point,. Proceedings of the 19th annual conference on Computer graphics and interactive techniques. 26(2), 71-78 (1992)

Jiao, X., H. Edelsabrunner y M.T. Heath, Mesh association formulation and algorithms, Proceedings of 8th International Meshing Roundtable, California, USA, 75-82 (1999)

Mahadevan, S., H. Pandzo, M. Bennamoun y J.A. Williams, A 3D acquisition and modelling system, Acoustic, Speech and Signal. Proceedings, (ICASSP'01). IEEE International Conference on, 3, 1941-1944 (2001).

Mencl., R., Reconstruction of surfaces from unrecognized 3D point clouds. Phd Thesis (2001).

Mori, A., T. Nakajima, T. Kaneko, H. Sakuma y Y. Aoki, Analysis of 109 Japanese children's lip and nose shapes using 3-dimensional digitizer, British journal of plastic surgery, 58, 318 (2005).

Natarajan, V. y H. Edelsbrunner, Simplification of three-dimensional density maps, IEEE Transactions on. Visualization Computer Graphics, 10(5), 587-597 (2004).

Pahole, I., I. Drstvensek, M. Ficko y J. Balic, Rapid prototyping processes give new possibilities to numerical copying techniques, 2005, Journal of Materials Processing Technology, 164-165, 1416-1422 (2005).

Remondino F., From point cloud to surface: the modelling and visualization problem. International workshop on visualization and animation of reality-based 3d models, 34, (2003).

Remondino, F. y A. Roditakis, Human figure reconstruction and modelling from single image or monocular video sequence, $4^{\text {th }}$ International Conference of 3D Digital Imaging and Modeling (3DIM). Canada (2003)

Yang, C,, Y. y G. Medioni, Object Modelling by registration of multiple range images, Image and Vision Computing: 10(3), 145-155 (1992). 